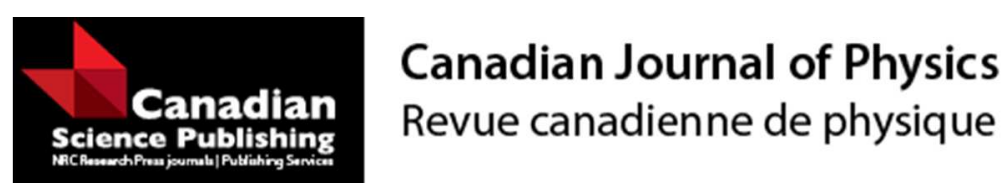

\title{
Anisotropic universe and thermodynamical illustration of the interacting generalized ghost dark energy
}

\begin{tabular}{|r|l|}
\hline Journal: & Canadian Journal of Physics \\
\hline Manuscript ID & cjp-2015-0821.R1 \\
\hline Manuscript Type: & Article \\
\hline Date Submitted by the Author: & 28-Feb-2016 \\
\hline Complete List of Authors: & Fayaz, V.; Islamic Azad University, Hamedan Branch, Physics Department \\
\hline Keyword: & $\begin{array}{l}\text { Anisotropic universe, Generalized ghost dark energy, Interacting dark } \\
\text { energy, Thermodynamic, ghost dark energy }\end{array}$ \\
\hline
\end{tabular}

SCHOLARONE $^{\text {tu }}$
Manuscripts 


\title{
Anisotropic universe and thermodynamical illustration of the interacting generalized ghost dark energy
}

\author{
V. Fayaz ${ }^{1, *}$ \\ ${ }^{1}$ Department of Physics, Hamedan Branch, Islamic Azad university, Hamedan, Iran
}

(Dated: March 8, 2016)

\begin{abstract}
We describe the thermodynamical interpretation of the interaction between ghost and general ghost dark energy and dark matter in a non-isotropic universe. At first, the presence/absence of interaction between ghost dark energy (GDE) and dark matter in both flat and non-isotropic geometry is discussed. Then, this investigation was extended to the noninteraction and interacting general ghost dark energy (GGDE) in a non-isotropic universe. Additionally, probable relation between the thermal fluctuations of the system and the assumed mutual interaction is investigated. Finally, we show that if one wants to solve the coincidence problem by using this mutual interaction, then the coupling constants of the interaction will be constrained. We extend our procedure to the first law of thermodynamics at apparent horizon of a BI universe, and assuming that the associated entropy with apparent horizon has a quantum corrected relation. Our study shows that, with the local equilibrium assumption, the generalized second law of thermodynamics is fulfilled in a region enclosed by the apparent horizon.
\end{abstract}

PACS Nos.: 95.36.+x, 95.35.+d, 98.80.-k

keywords: Anisotropic universe-Ghost dark energy-Generalized ghost dark energy-Interacting dark energy-Thermodynamic

\section{INTRODUCTIONS}

In relativistic cosmology, the cosmic acceleration observed can be described by a perfect fluid in which pressure $p$ and energy density $\rho$ satisfy the relation $\rho+3 p<0$. This kind of fluid with negative pressure is dubbed as Dark Energy (DE). Practically, the relation $\rho+3 p<0$ implies

\footnotetext{
${ }^{*}$ Electronic address: fayaz_vahid@yahoo.com
} 
that the EoS parameter $\omega$ (defined as $p / \rho$ ) must obey the condition $\omega<-1 / 3$, while, from an observational point of view, it is a difficult task to constrain its exact value [1-4]. The largest amount of the total cosmic energy density $\rho_{\text {tot }}$ is contained in the dark sectors, i.e. Dark Energy (DE) and Dark Matter (DM) which represent, respectively, about $70 \%$ and $25 \%$ of total energy density of the present day universe [5] while the ordinary baryonic matter we can observe with scientific instruments contributes for only the $5 \%$ of $\rho_{\text {tot }}$. Moreover, radiation contributes to the total cosmic energy density in a practically negligibly way. Usually, DE models are based on the scalar fields minimally coupled to gravity, as well as a possible coupling of the field to a background fluid (dark matter). Also, a possible coupling between the scalar field and the Ricci scalar is not to be excluded in the context of generalized Einstein gravity theories. For example, a quintessence field coupled to gravity and called "extended quintessence" was proposed in $[4,5]$. K-essence models non-minimally coupled to gravity were studied in $[6,7]$. It is also important to note that the first application of the non-minimal coupling to inflationary cosmology was done in literature [8]. Several different candidates have been studied in order to try to explain the DE nature, some of them include tachyon, quintessence, k-essence, quintom, Chaplygin gas, Agegraphic DE (ADE) and phantom [9-11, 13-15] (see [16, 17] for recent works on DE models).

Recently, a new kind of dark energy model, so called QCD ghost dark energy (GDE) model has been proposed [18]. This model, although is un-physical in the usual Minkoski space-time, express important physical effects in dynamical space-time or space-time with non-trivial topology. Actually, introducers of this dark energy model, claim the vacuum energy of Veneziano ghost flied in effective quantum field theory at low energy, is related to density of dark energy which is used for explaining the positive accelerating expansion of the universe $[19,20]$. They have shown that in a curved space time, the ghost field gives rise to a vacuum energy density $H \Lambda_{Q C D}^{3}$ of the right magnitude $\sim\left(10^{-3} \mathrm{eV}\right)^{4}$, where $H$ is the Hubble parameter and $\Lambda^{3}$ is QCD mass scale [21-25]. In [26], the author discussed that the contribution of the Veneziano QCD ghost field to the vacuum energy is not exactly of order $H$ and a subleading term $H^{2}$ appears due to the fact that the vacuum expectation value of the energy-momentum tensor is conserved in isolation [27]. This term can also lead to a better agreement with observations [28, 29]. Most discussions on dark energy rely on the assumption that it evolves independently of dark matter. Given the unknown nature of both dark energy and dark matter there is nothing in principle against their mutual interaction and it seems very special that these two major components in the universe are entirely independent. Indeed, this possibility has received a lot of attention recently (see [17-20] and references therein). In particular, it has been shown that the coupling can alleviate the coincidence problem [21]. 
Thermodynamic description of the Einstein equations is pointed out by Jacobson [30]. In order to generalize the Jacobson's results to the cosmological setup, we need to find a causal boundary for a spatially homogeneous FRW spacetime. In fact, it was argued that apparent horizon can be considered as a causal boundary for dynamical spacetimes [31-33]. In refs. [34, 35], the apparent horizon of the marginally trapped surface located is defined as $r_{A}=\frac{1}{\sqrt{H^{2}+k / a^{2}}}$. It was shown that the apparent horizon can be considered as a causal boundary for a spatially homogeneous FRW spacetime associated with gravitational entropy and surface gravity, and makes the establishment of the first law of thermodynamics [36-40]. The tendency of the universe to rise its entropy can be interpret as an origin for the gravity and thus the Einstein equations [41]. Therefore, the tendency of the cosmos, as a closed system, to increase its entropy is compatible with the current accelerated phase of the expansion. It was shown that for an accelerating universe the apparent horizon is a physical boundary from the thermodynamical point of view [42]. In particular, it was argued that for an accelerating universe inside the event horizon the generalized second law does not satisfy, while the accelerating universe enveloped by the apparent horizon satisfies the generalized second law of thermodynamics $[43,44]$. Therefore, the event horizon in an accelerating universe might not be a physical boundary from the thermodynamical point of view. Das et al. [45] showed that logarithmic corrections to the equilibrium thermodynamic entropy arise in all thermodynamic systems when stable fluctuations around equilibrium are taken into account and that, in particular, it leads to logarithmic corrections to the Bekenstein-Hawking formula for black hole entropy.

A Bianchi type I model, being the straightforward generalization of the flat FRW model, is one of the simplest models of the anisotropic universe that describes a homogeneous and spatially flat universe. For studying the possible effects of anisotropy in the early universe based on the present day observations many researchers [46-48] have investigated Bianchi type I models from different point of view. Recently, [49] have shown the importance of BI model to discuss the effects of anisotropy on the basis of recent evidences. Fayaz et al. [50] discussed generalized ghost dark energy for anisotropic cosmological models in Brans-Dicke theory. Hossienkhani et al. [51] investigated accelerating of the universe for Bianchi models in $f(R, T)$. We will study thermodynamics of the GDE and GGDE models when the interaction with DM is considered. We derive thermodynamic interpretations for such interactions in an anisotropic universe.

The paper is organized as follows. In the next section, we present the GDE scenario in the presence/absence of interaction between DE and DM in the BI universe and study its thermodynamics. We investigate the thermodynamics of the non-interacting GGDE in BI universe in Sect. III. In Sect. IV, we provide a thermodynamical interpretation for the GGDE interacting with DM. In 
Sect. V, we shall summarize the procedure constructed to study the thermodynamic properties of the apparent horizon through the non-interacting GGDE of a BI universe. The last section is devoted to summary and conclusions.

\section{METRIC AND GHOST DARK ENERGY MODEL IN BI UNIVERSE}

Let us first review the ghost dark energy model in flat Bianchi type I (BI) universe. Now, we intend to categorize our study into two types of the gas, i.e. noninteracting and interacting gas.

\section{A. Thermodynamical description of the noninteracting GDE}

The line element in our model is given by a Bianchi type I metric as

$$
d s^{2}=d t^{2}-A^{2}(t) d x^{2}-B^{2}(t) d y^{2}-C^{2}(t) d z^{2},
$$

where $A(t), B(t)$ and $C(t)$ are functions of cosmic time only. The contribution of the interaction with the matter fields is given by the energy momentum tensor which, is this case, is defined as

$$
T_{\nu}^{\mu}=\operatorname{diag}[\rho,-\omega \rho,-\omega \rho,-\omega \rho]
$$

where $\rho$ and $\omega$ represent the energy density and EoS parameter respectively. Einstein's field equations for BI metric given in (1) lead to the following system of equations [52]:

$$
\begin{aligned}
3 H^{2}-\sigma^{2} & =\frac{1}{M_{p}^{2}}\left(\rho_{m}+\rho_{\Lambda}\right), \\
3 H^{2}+2 \dot{H}+\sigma^{2} & =-\frac{1}{M_{p}^{2}}\left(p_{m}+p_{\Lambda}\right), \\
\dot{\sigma}+\theta \sigma & =0,
\end{aligned}
$$

where $M_{p}^{2}=1 /(8 \pi G), \rho_{\Lambda}$ and $p_{\Lambda}$ are the Planck mass, the energy density and pressure of dark energy, respectively, and $a=(A B C)^{\frac{1}{3}}$ is the scale factor, and $\sigma^{2}=1 / 2 \sigma_{i j} \sigma^{i j}$ in which $\sigma_{i j}=$ $u_{i, j}+\frac{1}{2}\left(u_{i ; k} u^{k} u_{j}+u_{j ; k} u^{k} u_{i}\right)+\frac{1}{3} \theta\left(g_{i j}+u_{i} u_{j}\right)$ is the shear tensor, which describes the rate of distortion of the matter flow, and $\theta=3 H=u_{; j}^{j}$ is the scalar expansion, where $u^{j}$ is 4 -velocity. In a co-moving coordinate system, i.e. $\left(u^{i}=\delta_{0}^{i}\right)$. Note that the model is considered to pressureless $\mathrm{DM}\left(p_{m}=0\right)$.

We investigate the GDE model in the framework of Einstein gravity. The GDE density is given by $[18,53]$

$$
\rho_{\Lambda}=\alpha H,
$$


where $\alpha$ is a constant with dimension $[\text { energy }]^{3}$, and roughly of order of $\Lambda_{Q C D}^{3}$ where $\Lambda_{Q C D} \sim$ $100 \mathrm{MeV}$. Using (3), the dimensionless density parameter can also be defined as usual

$$
\begin{aligned}
& \Omega_{m}=\frac{\rho_{m}}{\rho_{c r}}, \\
& \Omega_{\Lambda}=\frac{\rho_{\Lambda}}{\rho_{c r}}=\frac{\alpha}{3 M_{p}^{2} H}, \\
& \Omega_{\sigma}=\frac{\sigma^{2}}{3 H^{2}},
\end{aligned}
$$

where the critical energy density is $\rho_{c r}=3 M_{p}^{2} H^{2}$. Thus, the Bianchi equation can be rewritten as

$$
\Omega_{m}+\Omega_{\Lambda}=1-\Omega_{\sigma}
$$

Through this section we consider GDE in the absence of the interaction term, thus DE and DM evolves independent of each other and hence they satisfy the following conservation equations

$$
\begin{array}{r}
\dot{\rho}_{\Lambda}+3 H \rho_{\Lambda}\left(1+\omega_{\Lambda}^{0}\right)=0, \\
\dot{\rho}_{m}+3 H \rho_{m}=0 .
\end{array}
$$

Here $p_{\Lambda}=\rho_{\Lambda} \omega_{\Lambda}^{0}$ where $\omega_{\Lambda}^{0}$ is the equation of state parameter of the non-interacting GDE, and superscript (0) is used to remember that the GDE does not interact with DM. Taking the time derivative of relation (6) and using the $\mathrm{BI}$ equation we find

$$
\dot{\rho}_{\Lambda}=\rho_{\Lambda} \frac{\dot{H}}{H}=-\frac{\alpha}{2 M_{p}^{2}}\left[2 M_{p}^{2} \sigma^{2}+\rho_{\Lambda}\left(1+\omega_{\Lambda}^{0}+r\right)\right]
$$

where $r=\rho_{m} / \rho_{\Lambda}$ is the energy density ratio. Inserting this relation in continuity equation (6) we reach

$$
\left(1+\omega_{\Lambda}^{0}\right)\left(6 M_{p}^{2} H-\alpha\right)=\alpha r+2 \frac{M_{p}^{2} \sigma^{2}}{H}
$$

Substituting $\dot{\rho}_{\Lambda}$ into Eq. (6), after some simplifications, we find the EoS parameter of the GDE as

$$
\omega_{\Lambda}^{0}=\frac{-1+\Omega_{\sigma}^{0}}{2-\Omega_{\Lambda}^{0}}
$$

Using Eq. (13) and definition $\Omega_{\Lambda}$ in (8) we obtain

$$
\frac{\dot{H}}{H^{2}}=-3\left[\frac{\Omega_{\Lambda}^{0}}{2}\left(1+r+\omega_{\Lambda}^{0}\right)+\Omega_{\sigma}^{0}\right] .
$$

Taking the time derivative of $\Omega_{\Lambda}$ in Eq. (8), we find

$$
\dot{\Omega}_{\Lambda}=-\frac{\alpha}{3 M_{p}^{2}}\left(\frac{\dot{H}}{H^{2}}\right) .
$$


Using relation $\dot{\Omega}_{\Lambda}=H \Omega_{\Lambda}^{\prime}$, we obtain

$$
\Omega_{\Lambda}^{\prime}=\frac{3 \Omega_{\Lambda}^{0}}{2-\Omega_{\Lambda}^{0}}\left(1-\Omega_{\Lambda}^{0}+\Omega_{\sigma}^{0}\right)
$$

This is the equation of motion governing the evolution of GDE. Since the apparent horizon is the causal boundary of the BI spacetime, we write the first law of thermodynamics on the apparent horizon in order to find an expression for the entropy of the GDE as

$$
T d S_{\Lambda}=d E_{\Lambda}+p_{\Lambda} d V
$$

In this equation, $S_{\Lambda}$ is the entropy associated to the GDE while $V=4 \pi / 3 r_{A}^{3}$ and $E_{\Lambda}=\rho_{\Lambda} V$ are, respectively, the volume of the flat BI universe and the total energy of the GDE. Note that in flat $\mathrm{BI}$ universe the apparent horizon radius is indeed the Hubble radius, $r_{A}=1 / H$. The temperature $T$ of the GDE which will be equal to the temperature of the apparent horizon when thermodynamic equilibrium is supposed, and therefore we have

$$
T=\frac{1}{2 \pi r_{A}^{0}}=\frac{H_{0}}{2 \pi} .
$$

Therefore, for the volume and the total energy we have

$$
\begin{aligned}
& V=\frac{4 \pi}{3}\left(r_{A}^{0}\right)^{3}=\frac{4 \pi}{3} H_{0}^{-3}, \\
& E_{\Lambda}=\frac{4 \pi}{3} \alpha\left(r_{A}^{0}\right)^{2}=\frac{4 \pi}{3} \alpha H_{0}^{-2} .
\end{aligned}
$$

Differentiating relations (21) and (22) and substituting the results into (19), we get

$$
d S_{\Lambda}^{0}=8 \pi^{2} \alpha\left(r_{A}^{0}\right)^{2}\left(\frac{2}{3}+\omega_{\Lambda}^{0}\right) d r_{A}^{0},
$$

where we have used $p_{\Lambda}=\rho_{\Lambda} \omega_{\Lambda}^{0}$. Combining (23) with (15), we obtain

$$
d S_{\Lambda}^{0}=8 \pi^{2} \alpha\left(r_{A}^{0}\right)^{2} d r_{A}^{0}\left(\frac{2}{3}+\frac{-1+\Omega_{\sigma}^{0}}{2-\Omega_{\Lambda}^{0}}\right)
$$

for the ghost DE entropy when it is not coupled to DM. (A zero superscript or subscript indicates absence of interaction).

\section{B. Thermodynamical description of the interacting GDE}

We further assume the DE interact with DM. The recent observational evidence provided by the galaxy clusters also supports the interaction between dark energy and dark matter [54-58]. In the presence of interaction, the continuity equations are

$$
\begin{array}{r}
\dot{\rho}_{\Lambda}+3 H \rho_{\Lambda}\left(1+\omega_{\Lambda}\right)=-Q, \\
\dot{\rho}_{m}+3 H \rho_{m}=Q,
\end{array}
$$


where $Q$ represents the interaction term and we take it as

$$
Q=3 b^{2} H \rho_{\Lambda}(1+r)
$$

with $b^{2}$ being a coupling constant. It should be noted that the ideal interaction term must be motivated from the theory of quantum gravity. In the absence of such a theory, we rely on pure dimensional basis for choosing an interaction $Q$. In the present work for the sake of generality, we choose the third expression for the interaction term. Inserting Eqs. (13) and (27) in Eq. (25) and using $r=\left(1-\Omega_{\sigma}-\Omega_{\Lambda}\right) / \Omega_{\Lambda}$ we find

$$
\omega_{\Lambda}=\frac{-1+\Omega_{\sigma}}{2-\Omega_{\Lambda}}\left(1+\frac{2 b^{2}}{\Omega_{\Lambda}}\right) .
$$

One can easily check that in the late time where $\Omega_{\Lambda} \rightarrow 1$ and $\Omega_{\sigma} \rightarrow 0$, the equation of state parameter of interacting ghost dark energy necessary crosses the phantom line, namely, $\omega_{\Lambda}=$ $-\left(1+2 b^{2}\right)<-1$ independent of the value of coupling constant $b^{2}$. Inserting (6) and (10) into the (27), we find

$$
b^{2}=\frac{Q \Omega_{\Lambda}}{3 \alpha H^{2}\left(1-\Omega_{\sigma}\right)}
$$

which yields

$$
\omega_{\Lambda}=-\frac{1}{2-\Omega_{\Lambda}}\left(1-\Omega_{\sigma}+\frac{2 Q r_{A}^{2}}{3 \alpha}\right)
$$

The evolution behavior of the ghost dark energy is now given by

$$
\Omega_{\Lambda}^{\prime}=\frac{3}{2} \Omega_{\Lambda}\left[1+\Omega_{\sigma}+\frac{\Omega_{\Lambda}\left(-1+\Omega_{\sigma}\right)}{2-\Omega_{\Lambda}}\left(1+\frac{2 b^{2}}{\Omega_{\Lambda}}\right)\right] .
$$

The evolution of $\Omega_{\Lambda}$ and $\omega_{\Lambda}$ are shown in Fig. 1 for different interacting parameter $b^{2}$. It clear
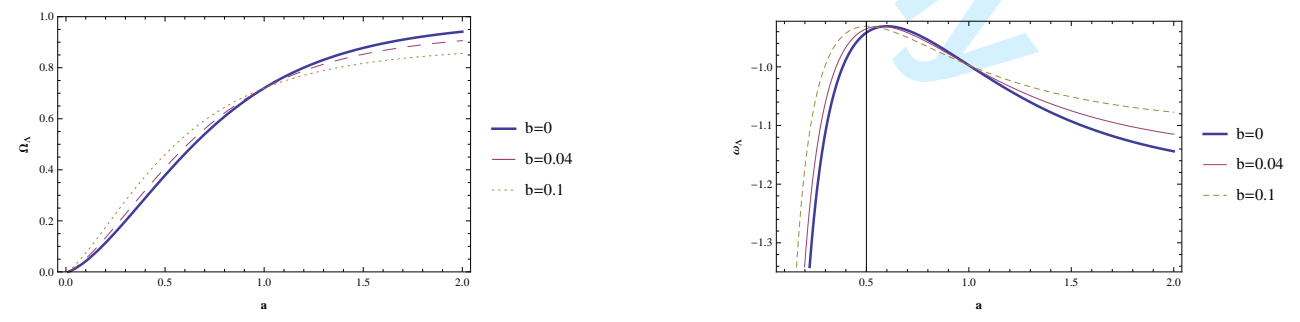

(a)

FIG. 1: Left panel corresponds to of the GDE density parameter $\Omega_{\Lambda}$ with different interacting parameter $b^{2}$ while right panel shows the evolution of $\omega_{\Lambda}$. Here we have taken $\Omega_{\Lambda}^{0}=0.69$ and $\Omega_{\sigma}=0.001$.

that the EoS parameter decrease with the scale factor increase and it crosses the phantom divide 
line in the presence interacting DM and DE. Comparing (30) with (15), we see that the presence of the interaction term $Q$ has provoked a change in the equation of state parameter and consequently in the dimensionless density parameter of the dark energy component and thus now there is no subscript above the aforesaid quantities to denote the absence of interaction. The interacting GDE model in the non-isotropic universe as described above is not anymore thermodynamically interpreted as a state in thermodynamical equilibrium. Indeed, as soon as an interaction between dark components is taken account, they cannot remain in their respective equilibrium states. The effect of interaction between the dark components is thermodynamically interpreted as a small fluctuation around the thermal equilibrium. It was shown [45] that due to the fluctuation, there is a leading logarithmic correction $S_{\Lambda}^{1}=-\frac{1}{2} \ln \left(C^{\prime} T^{2}\right)$ to the thermodynamic entropy around equilibrium in all thermodynamical systems. In order to find a relation between thermal fluctuations and the dark sectors mutual interaction, we express the entropy of the interacting GDE model as

$$
S_{\Lambda}=S_{\Lambda}^{0}+S_{\Lambda}^{1}+S_{\Lambda}^{2}
$$

In the above equation, $S_{\Lambda}^{0}$ and $S_{\Lambda}^{1}$ are the entropy of GDE, when there is no mutual interaction between the dark sectors of the universe, and logarithmic correction to the entropy, which is due to the thermal fluctuations, respectively. $S_{\Lambda}^{2}$ also concerns higher order terms which are so week in the gravitational and cosmological systems [45]. We should note that since the $S_{\Lambda}^{0}$ and $S_{\Lambda}^{1}$ terms have the major contributions in the expression (32), one can withdraw the $S_{\Lambda}^{2}$ contribution, and writes $S_{\Lambda}=S_{\Lambda}^{0}+S_{\Lambda}^{1}$. Moreover, $C^{\prime}=T \frac{\partial S_{\Lambda}^{0}}{\partial T}$ is the dimensionless heat capacity, and it is also useful to mention again that this analysis is valid for all thermodynamical systems [45]. It is a matter of calculation to show that

$$
C^{\prime}=T \frac{\partial S_{\Lambda}^{0}}{\partial T}=-8 \pi^{2} \alpha\left(r_{A}^{0}\right)^{3}\left(\frac{2}{3}+\frac{-1+\Omega_{\sigma}^{0}}{2-\Omega_{\Lambda}^{0}}\right)
$$

which is positive since for ghost DE one has $\frac{2}{3}+\frac{-1+\Omega_{\sigma}^{0}}{2-\Omega_{\Lambda}^{0}}<0$. Accordingly, the fluctuation is indeed stable and the entropy correction read

$$
S_{\Lambda}^{1}=-\frac{1}{2} \ln \left(-2 \alpha r_{A}^{0}\left(\frac{2}{3}+\frac{-1+\Omega_{\sigma}^{0}}{2-\Omega_{\Lambda}^{0}}\right)\right) .
$$

Using (23), (30) and (32) can be expressed in terms of interaction term as

$$
Q=\frac{\alpha\left(2-\Omega_{\Lambda}\right)}{\left(r_{A}\right)^{2}}+\frac{3 \alpha\left(-1+\Omega_{\sigma}\right)}{2\left(r_{A}\right)^{2}}-\frac{3\left(2-\Omega_{\Lambda}\right)}{16 \pi^{2}\left(r_{A}\right)^{4}}\left(\frac{d S_{\Lambda}^{0}}{d r_{A}}+\frac{d S_{\Lambda}^{1}}{d r_{A}}\right),
$$

where

$$
\frac{d S_{\Lambda}^{0}}{d r_{A}}=\frac{\partial S_{\Lambda}^{0}}{\partial r_{A}^{0}} \frac{d r_{A}^{0}}{d r_{A}}=8 \pi^{2} \alpha\left(r_{A}^{0}\right)^{2}\left(\frac{2}{3}+\frac{-1+\Omega_{\sigma}^{0}}{2-\Omega_{\Lambda}^{0}}\right) \frac{d r_{A}^{0}}{d r_{A}}
$$


and

$$
\frac{d S_{\Lambda}^{1}}{d r_{A}}=\frac{\partial S_{\Lambda}^{1}}{\partial r_{A}^{0}} \frac{d r_{A}^{0}}{d r_{A}}=-\frac{1}{2 r_{A}^{0}} \frac{d r_{A}^{0}}{d r_{A}} .
$$

Finally, inserting $r_{A}=1 / H$ and $r_{A}^{0}=1 / H_{0},(35)$ takes the form

$$
\begin{aligned}
& Q=\alpha H^{2}\left(2-\Omega_{\Lambda}\right)+\frac{3}{2} \alpha H^{2}\left(-1+\Omega_{\sigma}\right)+\left(2-\Omega_{\Lambda}\right) H^{4} \\
& \times\left[-\frac{\alpha}{H_{0}^{2}}\left(1+\frac{3}{2}\left(\frac{-1+\Omega_{\sigma}^{0}}{2-\Omega_{\Lambda}^{0}}\right)\right)+\frac{3 H_{0}}{32 \pi^{2}}\right] \frac{d r_{A}^{0}}{d r_{A}},
\end{aligned}
$$

which is an expression for the interaction between the GDE and DM components of the BI universe. In order to solve the coincidence problem, DE should decay into the DM sector meaning that $Q>0$ [40]. Therefore, the permissible thermal fluctuations are those leading to $Q>0$ and vice versa. The latter means that, when the mutual interaction between the dark sectors meets the $Q>0$ condition, then it leaves a physically acceptable fluctuation into the system. Since we have considered interactions in the $Q=3 b^{2} H\left(\rho_{m}+\rho_{\Lambda}\right)$ form, the $Q>0$ condition leads to $b^{2}>0$. In order to investigate the validity of this criterion, we use Eq. (28)

$$
\frac{2 b^{2}}{\Omega_{\Lambda}}+1=\frac{2-\Omega_{\Lambda}}{-1+\Omega_{\sigma}} \omega_{\Lambda}
$$

Because the LHS of this equation is positive and $0<\Omega_{\Lambda}<1$ and $\Omega_{\sigma} \simeq 0.001$ for present time, $\omega_{\Lambda}$ should meet the $\omega_{\Lambda}<0$ condition. Loosely speaking, when the state parameter of DE meets the $\omega_{\Lambda}<0$ condition, its mutual interaction with DM may lead to solve the coincidence problem and induces thermal fluctuations into the system in accordance with (38). In this way we provide a relation between the dark components interaction and the thermal fluctuations around the equilibrium state.

\section{THERMODYNAMICAL DESCRIPTION OF THE NONINTERACTING GGDE IN BI}

Here, we consider the flat FRW universe filled by the GGDE and the DM whereas the dark sectors do not interact with each other. Therefore, the generalized ghost energy density may be written as $[28]$

$$
\rho_{\Lambda}=\alpha H+\beta H^{2}
$$

where $\beta$ is a constant. In the original $\operatorname{GDE}(\beta=0)$ with $\Lambda_{Q C D} \sim 100 \mathrm{MeV}$ and $H \sim 10^{-33} \mathrm{eV}$, $\Lambda_{Q C D}^{3} H$ gives the right order of magnitude $\sim\left(3 \times 10^{3} \mathrm{eV}\right)^{4}$ for the observed DE density [18]. In 
the GGDE, $\beta$ is a free parameter and can be adjusted for better agreement with observations. In addition, for the dimensionless density parameter of the GGDE we obtain

$$
\Omega_{\Lambda}=\frac{\rho_{\Lambda}}{\rho_{c r}}=\frac{\alpha+\beta H}{3 M_{p}^{2} H}
$$

Combining relations (13) and $\dot{\rho}_{\Lambda}=\dot{H}(\alpha+2 \beta H)$ with non-interacting continuity equation (11), we get

$$
\left(1+\omega_{\Lambda}^{0}\right)\left[3 H-\frac{1}{2 M_{p}^{2}}(\alpha+2 \beta H)\right]=\frac{1}{2 M_{p}^{2} \Omega_{\Lambda}^{0}}\left(1+\Omega_{\sigma}^{0}-\Omega_{\Lambda}^{0}\right)(\alpha+2 \beta H) .
$$

Solving the above equation for $\omega_{\Lambda}$ and noticing that $r=\Omega_{m} / \Omega_{\Lambda}$, we find

$$
\omega_{\Lambda}^{0}=\frac{\xi-\Omega_{\Lambda}^{0}+\Omega_{\sigma}^{0}\left(\Omega_{\Lambda}^{0}+\xi\right)}{\Omega_{\Lambda}^{0}\left(2-\Omega_{\Lambda}^{0}-\xi\right)}
$$

where $\xi=\beta /\left(3 M_{p}^{2}\right)$. Setting $\xi=0, \omega_{\Lambda}$ reduces to the respective relation in the absence of interaction obtained in (15). When $\xi=\sigma=0$ the result recovers those in [29] for original GDE. We can also study the evolution behavior of the GGDE. For this, differentiating Eq. (41) with respect to time and using Eqs. (11), (16) and (40) and relation $\dot{\Omega}_{\Lambda}=H \Omega_{\Lambda}^{\prime}$, we obtain

$$
\Omega_{\Lambda}^{\prime}=-\left(\Omega_{\Lambda}^{0}-\xi\right) \frac{\dot{H}}{H^{2}}=3\left(\frac{\left(1-\Omega_{\Lambda}^{0}+\Omega_{\sigma}^{0}\right)\left(\Omega_{\Lambda}^{0}-\xi\right)}{2-\Omega_{\Lambda}^{0}-\xi}\right) .
$$

In order to find the entropy changes of the GGDE, we assume that the first law of thermodynamics is available on the apparent horizon, where the pressure of the GGDE is

$$
p_{\Lambda}=\rho_{\Lambda} \omega_{\Lambda}^{0}=H_{0}\left(\alpha+\beta H_{0}\right) \omega_{\Lambda}^{0}=\frac{1}{r_{A}^{0}}\left(\alpha+\frac{\beta}{r_{A}^{0}}\right) \omega_{\Lambda}^{0},
$$

and following the recipe of Sect. IIA, we reach

$$
d S_{\Lambda}^{0}=8 \pi^{2} r_{A}^{0} d r_{A}^{0}\left[\frac{1}{3}\left(2 r_{A}^{0} \alpha+\beta\right)+\left(r_{A}^{0} \alpha+\beta\right) \frac{\xi-\Omega_{\Lambda}^{0}+\Omega_{\sigma}^{0}\left(\Omega_{\Lambda}^{0}+\xi\right)}{\Omega_{\Lambda}^{0}\left(2-\Omega_{\Lambda}^{0}-\xi\right)}\right] .
$$

Again, the subscript/superscript (0) indicates that the GGDE and the DM do not interact with each other.

\section{THERMODYNAMICAL DESCRIPTION OF THE INTERACTING GGDE IN BI}

In the previous section, the evolution of the DE and DM components were discussed separately. Here we extend the study to the interacting case, seeking new features of GGDE. Inserting Eqs. (27) and relation $\dot{\rho}_{\Lambda}=\dot{H}(\alpha+2 \beta H)$ in (25) and using $1+r=\left(1-\Omega_{\sigma}\right) / \Omega_{\Lambda}$ we find

$$
\omega_{\Lambda}=\frac{\xi-\Omega_{\Lambda}+\Omega_{\sigma}\left(\Omega_{\Lambda}+\xi\right)-2 b^{2}\left(1-\Omega_{\sigma}\right)}{\Omega_{\Lambda}\left(2-\Omega_{\Lambda}-\xi\right)},
$$


Now, since $Q=3 b^{2} H \rho_{\Lambda}(1+r)$ we get

$$
b^{2}=\frac{Q \Omega_{\Lambda}}{3 H^{2}\left(1-\Omega_{\sigma}\right)(\alpha+\beta H)},
$$

Replacing this relation into (47) one obtains

$$
\omega_{\Lambda}=\frac{\xi-\Omega_{\Lambda}+\Omega_{\sigma}\left(\Omega_{\Lambda}+\xi\right)-\frac{2 Q \Omega_{\Lambda}}{\frac{3}{r_{A}^{2}\left(\alpha+\frac{\beta}{r_{A}}\right)}}}{\Omega_{\Lambda}\left(2-\Omega_{\Lambda}-\xi\right)} .
$$

It is obvious that, the results of sections III and IIB are available in the $Q \rightarrow 0$ and $\beta \rightarrow 0$ limits respectively. Using relation (40) as well as (47), it is a matter of calculation to show

$$
\Omega_{\Lambda}^{\prime}=-\left(\Omega_{\Lambda}-\xi\right) \frac{\dot{H}}{H^{2}}=3\left(\Omega_{\Lambda}-\xi\right) \frac{1-\Omega_{\Lambda}+\Omega_{\sigma}-b^{2}\left(1-\Omega_{\sigma}\right)}{2-\Omega_{\Lambda}-\xi} .
$$

For a better insight we have plotted $\Omega_{\Lambda}$ and $\omega_{\Lambda}$ against $a$ for differnt $b^{2}$ in Fig. 2 . It worth mentioning that at the late time where $a \gg$ and $\Omega_{\sigma} \rightarrow 0$ the effective EoS parameter approaches less than -1 , i.e. $\omega_{\Lambda}<-1$, which reminds a super acceleration for the universe in the future. Substituting Eq. (46) into (33) the heat capacity is given by
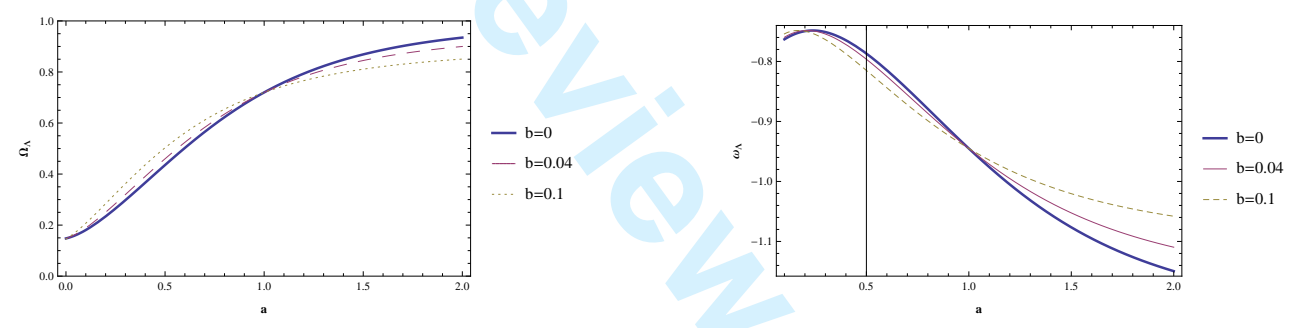

(a)

FIG. 2: In the first figure $\Omega_{\Lambda}$ is plotted against $a$ in the BI flat interacting GGDE. In the second figure the EoS parameter are plotted versus $a$ for $\xi=0.15, \Omega_{\Lambda}=0.69$ and $\Omega_{\sigma}=0.001$.

$$
C^{\prime}=8 \pi^{2}\left(r_{A}^{0}\right)^{2}\left[\frac{1}{3}\left(2 r_{A}^{0} \alpha+\beta\right)+\left(r_{A}^{0} \alpha+\beta\right) \frac{\xi-\Omega_{\Lambda}^{0}+\Omega_{\sigma}^{0}\left(\Omega_{\Lambda}^{0}+\xi\right)}{\Omega_{\Lambda}^{0}\left(2-\Omega_{\Lambda}^{0}-\xi\right)}\right] .
$$

Therefore, for the logarithmic correction of entropy due to the thermal fluctuation we reach

$$
S_{\Lambda}^{1}=-\frac{1}{2} \ln \left(-2\left[\frac{1}{3}\left(2 r_{A}^{0} \alpha+\beta\right)+\left(r_{A}^{0} \alpha+\beta\right) \frac{\xi-\Omega_{\Lambda}^{0}+\Omega_{\sigma}^{0}\left(\Omega_{\Lambda}^{0}+\xi\right)}{\Omega_{\Lambda}^{0}\left(2-\Omega_{\Lambda}^{0}-\xi\right)}\right]\right) .
$$

Considering the first law of thermodynamics and using (49), we get

$$
\frac{d S_{\Lambda}}{d r_{A}}=8 \pi^{2} r_{A}\left[\frac{1}{3}\left(2 r_{A} \alpha+\beta\right)+\left(r_{A} \alpha+\beta\right) \frac{\xi-\Omega_{\Lambda}+\Omega_{\sigma}\left(\Omega_{\Lambda}+\xi\right)-\frac{2 Q \Omega_{\Lambda}}{\frac{3}{r_{A}^{2}}\left(\alpha+\frac{\beta}{r_{A}}\right)}}{\Omega_{\Lambda}\left(2-\Omega_{\Lambda}-\xi\right)}\right] .
$$


Since $\frac{d S_{\Lambda}}{d r_{A}}=\frac{d S_{\Lambda}^{0}}{d r_{A}}+\frac{d S_{\Lambda}^{1}}{d r_{A}}$, we need to evaluate $\frac{d S_{\Lambda}^{0}}{d r_{A}}$ and $\frac{d S_{\Lambda}^{1}}{d r_{A}}$. Calculations lead to

$$
\frac{d S_{\Lambda}^{0}}{d r_{A}}=\left(8 \pi^{2} r_{A}^{0}\left[\frac{1}{3}\left(2 r_{A}^{0} \alpha+\beta\right)+\left(r_{A}^{0} \alpha+\beta\right) \frac{\xi-\Omega_{\Lambda}^{0}+\Omega_{\sigma}^{0}\left(\Omega_{\Lambda}^{0}+\xi\right)}{\Omega_{\Lambda}^{0}\left(2-\Omega_{\Lambda}^{0}-\xi\right)}\right]\right) \frac{d r_{A}^{0}}{d r_{A}}
$$

and

$$
\frac{d S_{\Lambda}^{1}}{d r_{A}}=\frac{\partial S_{\Lambda}^{1}}{\partial r_{A}^{0}} \frac{d r_{A}^{0}}{d r_{A}}=-\frac{1}{2 r_{A}^{0}} \frac{d r_{A}^{0}}{d r_{A}}
$$

Finally, we get

$$
\begin{aligned}
Q= & {\left[\frac{3\left(2-\Omega_{\Lambda}-\xi\right)}{32 \pi^{2} r_{A}^{4} r_{A}^{0}}-\frac{r_{A}^{0}}{2 r_{A}^{4} \Omega_{\Lambda}}\left(\beta+2 \alpha r_{A}^{0}+3\left(\beta+\alpha r_{A}^{0}\right)\left[\frac{\xi-\Omega_{\Lambda}^{0}+\Omega_{\sigma}^{0}\left(\Omega_{\Lambda}^{0}+\xi\right)}{\Omega_{\Lambda}^{0}\left(2-\Omega_{\Lambda}^{0}-\xi\right)}\right]\right)\right.} \\
& \left.+\frac{\beta+2 \alpha r_{A}}{2 r_{A}^{3}}\left(2-\Omega_{\Lambda}-\xi\right)\right] \frac{d r_{A}^{0}}{d r_{A}}+\frac{3\left(\beta+\alpha r_{A}\right)}{2 r_{A}^{3} \Omega_{\Lambda}}\left[\xi-\Omega_{\Lambda}+\Omega_{\sigma}\left(\Omega_{\Lambda}+\xi\right)\right] .
\end{aligned}
$$

Again, $r_{A}^{0}$ is the radius of the apparent horizon when the GGDE does not interact with the DM and therefore, there is no thermal fluctuations around the equilibrium state of the universe. In addition, $r_{A}$ is the radius of the universe accelerated by an interacting GGDE. In order to alleviate the coincidence problem we should have $b^{2}>0$. From (47) we get

$$
-2 b^{2}\left(1-\Omega_{\sigma}\right)=\left[\Omega_{\Lambda}\left(2-\Omega_{\Lambda}-\xi\right)\right] \omega_{\Lambda}-\xi+\Omega_{\Lambda}-\Omega_{\sigma}\left(\Omega_{\Lambda}+\xi\right)
$$

Since the LHS of this equation is negative, we should have $\left[\Omega_{\Lambda}\left(2-\Omega_{\Lambda}-\xi\right)\right] \omega_{\Lambda}-\xi+\Omega_{\Lambda}-\Omega_{\sigma}\left(\Omega_{\Lambda}+\right.$ $\xi)<0$ leading to the $\omega_{\Lambda}<\frac{\xi-\Omega_{\Lambda}+\Omega_{\sigma}\left(\Omega_{\Lambda}+\xi\right)}{\Omega_{\Lambda}\left(2-\Omega_{\Lambda}-\xi\right)}$ condition for $\omega_{\Lambda}$. Therefore, when the equation of state parameter of DE meets the $\omega_{\Lambda}<\frac{\xi-\Omega_{\Lambda}+\Omega_{\sigma}\left(\Omega_{\Lambda}+\xi\right)}{\Omega_{\Lambda}\left(2-\Omega_{\Lambda}-\xi\right)}$ condition, DE decays into DM in agreement with the solving of the coincidence problem leading to leave thermal fluctuations in the system. Finally, we should note that the (56) is indeed, a relation between the interaction and the thermodynamic fluctuations.

\section{THERMODYNAMICS BEHAVIOR OF NON-INTERACTING GGDE AT APPARENT HORIZON OF BI UNIVERSE}

It is interesting to note that Friedmann equations, in Einstein's gravity, can be derived by applying the Clausius relation to the apparent horizon of FRW universe, in which entropy is assumed to be proportional to its horizon area, $S=A / 4 G$ [59]. However, this definition for entropy can be modified from the inclusion of quantum effects, motivated from the loop quantum gravity (LQG). The corrected entropy takes the form [60]

$$
S_{\Lambda}=\frac{A}{4 G}-\alpha^{\prime} \ln \frac{A}{4 G}+\beta^{\prime} \frac{4 G}{A},
$$


where $\alpha^{\prime}$ and $\beta^{\prime}$ are positive dimensionless constants of order unity. Assuming the first law of thermodynamics on the apparent horizon is satisfied and has the form $d E_{\Lambda}=T d S_{\Lambda}+W_{\Lambda} d V$ with $W_{\Lambda}=\left(\rho_{\Lambda}-p_{\Lambda}\right) / 2$. The associated temperature with the apparent horizon can be defined as $T=\frac{\kappa}{2 \pi}$, where $\kappa$ is the surface gravity $\kappa=-\frac{1}{r_{A}}\left(1-\frac{r_{A}^{\prime}}{2 H r_{A}}\right)$. Taking differential form of the relation $E_{\Lambda}=\rho_{\Lambda} \frac{4 \pi}{3} r_{A}^{3}$ for the energy inside the apparent horizon, we get

$$
d E_{\Lambda}=4 \pi r_{A}^{2}\left(\rho_{\Lambda} d r_{A}+\frac{1}{3} r_{A} \dot{\rho}_{\Lambda} d t\right)
$$

Using the continuity equation (11), we obtain

$$
d E_{\Lambda}=4 \pi r_{A}^{2} \rho_{\Lambda}\left[d r_{A}-H r_{A}\left(1+\omega_{\Lambda}^{0}\right) d t\right]
$$

Taking differential form of the corrected entropy (58), we have

$$
d S_{\Lambda}=\frac{2 \pi r_{A}}{G}\left(1-\frac{\alpha^{\prime} G}{\pi r_{A}^{2}}-\frac{\beta^{\prime} G^{2}}{\pi^{2} r_{A}^{4}}\right) d r_{A},
$$

Inserting Eqs. (60) and (61) in the first law of thermodynamics on the apparent horizon $d E_{\Lambda}=$ $T d S_{\Lambda}+W_{\Lambda} d V$ and using the relation between temperature and surface gravity, we can get

$$
\frac{\dot{r}_{A}}{r_{A}^{3}}\left(1-\frac{\alpha^{\prime} G}{\pi r_{A}^{2}}-\frac{\beta^{\prime} G^{2}}{\pi^{2} r_{A}^{4}}\right)=-4 \pi G \rho_{\Lambda}\left(1+\omega_{\Lambda}^{0}\right) .
$$

Substituting Eqs. (40), (43) into (62), we find the expression for $\dot{r}_{A}$

$$
\dot{r}_{A}=4 \pi G H^{2} r_{A}^{3}(\alpha+\beta H) \frac{\left(\Omega_{\Lambda}^{0}+\xi\right)\left(1-\Omega_{\Lambda}^{0}+\Omega_{\sigma}^{0}\right)}{\Omega_{\Lambda}^{0}\left(2-\Omega_{\Lambda}^{0}-\xi\right)}\left[1-\frac{\alpha^{\prime} G}{\pi r_{A}^{2}}-\frac{\beta^{\prime} G^{2}}{\pi^{2} r_{A}^{4}}\right]^{-1} .
$$

One can see from the above equation that $\frac{\left(\Omega_{\Lambda}^{0}+\xi\right)\left(1-\Omega_{\Lambda}^{0}+\Omega_{\sigma}^{0}\right)}{\Omega_{\Lambda}^{0}\left(2-\Omega_{\Lambda}^{0}-\xi\right)}>0$ provided the derivative of apparent horizon radius, $\dot{r}_{A}>0$. It is important to note that the expression in the bracket of (63) is positive at the present time, i.e., $\left[1-\frac{\alpha^{\prime} G}{\pi r_{A}^{2}}-\frac{\beta^{\prime} G^{2}}{\pi^{2} r_{A}^{4}}\right]>0$. At the early time where $r_{A} \rightarrow 0$ the generalized second law of thermodynamics may be violated but in that case the local equilibrium hypothesis is failed too. Besides, from the physical point of view, the effect of the correction terms on the entropy should be less than uncorrected term. Thus, the second and third terms on the right hand side of Eqs. (58) and (61) should be much smaller than the first term, otherwise these terms cannot be regarded as the correction terms. Let us now turn to find out $T \dot{S}_{\Lambda}$ :

$$
T \dot{S}_{\Lambda}=\frac{1}{r_{A}}\left(1-\frac{\dot{r}_{A}}{2 H r_{A}}\right) \frac{d}{d t}\left(\frac{A}{4 G}-\alpha^{\prime} \ln \frac{A}{4 G}+\beta^{\prime} \frac{4 G}{A}\right)
$$

After some simplification and using Eq. (63) we obtain

$$
T \dot{S}_{\Lambda}=H(\alpha+\beta H) \frac{\left(\Omega_{\Lambda}^{0}+\xi\right)\left(1-\Omega_{\Lambda}^{0}+\Omega_{\sigma}^{0}\right)}{\Omega_{\Lambda}^{0}\left(2-\Omega_{\Lambda}^{0}-\xi\right)}\left(1-\frac{\dot{r_{A}}}{2 H r_{A}}\right)
$$


where $A=4 \pi r_{A}^{2}$ is the apparent horizon area. Using the fact that in flat Bi universe we have $\dot{r_{A}}=-\dot{H} / H$ and the deceleration parameter $q=-1-\dot{H} / H^{2}$, Eq. (65) can be written as

$$
T \dot{S}_{\Lambda}=\frac{1}{2} H(\alpha+\beta H) \frac{\left(\Omega_{\Lambda}^{0}+\xi\right)\left(1-\Omega_{\Lambda}^{0}+\Omega_{\sigma}^{0}\right)}{\Omega_{\Lambda}^{0}\left(2-\Omega_{\Lambda}^{0}-\xi\right)}(1-q) .
$$

For an accelerating universe we have $q<0$, and hence the right hand side of the above equation cannot be negative throughout the history of the universe, which means that $\dot{S}_{\Lambda}>0$ always holds.

\section{CONCLUSION}

Along the paper, the Bianchi type-I metric with GDE and GGDE scenario have been studied. We have investigated thermodynamic of GDE in the flat BI universe, and can be written directly in the form of the first law of thermodynamics, $d E=T d S+\rho d V$, at the apparent horizon. We obtained an expression for the equation of state parameter of the interacting GDE, as well as an expression for its entropy changes. We have disclosed the deep connection between the thermodynamics and GGDE by the apparent horizon. In continue, we got an expression for the equation of state parameter of the interacting GGDE as well as a relation between the thermal fluctuations and the mutual interaction of the GGDE and the pressureless DM. The corresponding limitations on the state parameter were derived for both of the GDE and GGDE models. Concisely, we think our survey shows that the interaction between the dark sectors of the universe can be considered as the cause of the thermal fluctuations and thus, the logarithmic correction to the entropy of the GDE and GGDE models. In the following, applying the first law of thermodynamics, $d E_{\Lambda}=T d S_{\Lambda}+W_{\Lambda} d V$, to apparent horizon of a BI universe and assuming that the apparent horizon has temperature $T=\frac{1}{2 \pi r_{A}}\left(1-\frac{r_{A}}{2 H r_{A}}\right)$, and a quantum corrected entropy-area relation, $S_{\Lambda}=$ $\frac{A}{4 G}-\alpha^{\prime} \ln \frac{A}{4 G}+\beta^{\prime} \frac{4 G}{A}$, we are able to derive BI equations governing the dynamical evolution of the universe. We have also investigated the validity of the generalized second law of thermodynamics for the BI universe.

\section{ACKNOWLEDGMENT}

I am grateful to the referee for valuable comments and suggestions, which have allowed us to improve this paper significantly.

[1] A.G. Riess, A.V. Filippenko, P. Challis, et al., Astron. J. 116, 1009 (1998). 
[2] S. Perlmutter, G. Aldering, G. Goldhaber, et al., Astrophys. J. 517, 565 (1999).

[3] D.N. Spergel et al., Astrophy. J. Suppl. 148, 175 (2003).

[4] M. Tegmark et al., Phys. Rev. D 69, 103501 (2004).

[5] H.V. Peiris et al., Astrophys. J. Suppl. Ser. 148, 213 (2003).

[6] R.C. de Souza, G.M. Kremer, Class. Quant. Grav. 26, 135008 (2009).

[7] A.A. Sen, N. Chandrachani Devi, Gen. Rel. Grav. 42, 821 (2010).

[8] B.L. Spokoiny, Phys. Lett. B 147, 39 (1984).

[9] B. Boisseau, G. Esposito-Farese, D. Polarski, A.A. Starobinsky, Phys. Rev. Lett. 85, 2236 (2000).

[10] H. Kleinert, H.J. Schmidt, Gen. Rel. Grav. 34, 1295 (2002).

[11] S. Capozziello, V.F. Cardone, S. Carloni, A. Troisi, Int. J. Mod. Phys. D 12, 1969 (2003).

[12] S. Nojiri, S.D. Odintsov, AIP Conf. Proc. 1115, 212 (2009).

[13] A. Sen, Mod. Phys. Lett. A 17, 1797 (2002).

[14] A. Sen, JHEP 10, 8 (1999).

[15] K. Saaidi, A. Aghamohammadi, Phys. Scripta 83, 025902 (2011).

[16] T. Padmanabhan, Phys. Rep. 380, 235, (2003).

[17] Y.F. Cai, E.N. Saridakis, M.R. Setare, J.Q. Xia, Phys. Rep. 493, 1 (2010).

[18] N. Ohta, Phys. Lett. B 695, 41 (2011).

[19] E. Witten, Nucl. Phys. B 156, 269 (1979).

[20] G. Veneziano, Nucl. Phys. B 159, 213 (1979).

[21] F.R. Urban and A.R. Zhitnitsky, Phys. Lett. B 688, 9 (2010).

[22] A.R. Zhitnitsky, Phys. Rev. D 82, 103520 (2010).

[23] B. Holdom, Phys. Lett. B 697, 351 (2011).

[24] A.R. Zhitnitsky, Phys. Rev. D 84, 124008 (2011).

[25] E. Thomas, A.R. Zhitnitsky, Phys. Rev. D 85, 044039 (2012).

[26] A.R. Zhitnitsky, Phys. Rev. D 86, 045026 (2012).

[27] M. Maggiore, Phys. Rev. D 83, 063514 (2011).

[28] R.G. Cai, Z.L. Tuo, Y.B. Wu, Y.Y. Zhao, Phys. Rev. D 86, 023511 (2012).

[29] A. Sheykhi, M. Sadegh Movahed, Gen Relativ Gravit 44, 449 (2012).

[30] T. Jacobson, Phys. Rev. Lett. 75, 1260 (1995).

[31] S.A. Hayward, S. Mukohyana, and M.C. Ashworth, Phys. Lett. A 256, 347 (1999).

[32] S.A. Hayward, Class. Quantum Grav. 15, 3147 (1998).

[33] D. Bak and S.J. Rey, Class. Quantum Grav. 17, L83 (2000).

[34] A. Sheykhi, B. Wang and R.G. Cai, Nucl. Phys. B 779, 1 (2007).

[35] A. Sheykhi, B. Wang and R.G. Cai, Phys. Rev. D 76, 023515 (2007).

[36] M. Akbar and R.G. Cai, Phys. Rev. D 75, 084003 (2007).

[37] R.G. Cai and L.M. Cao, Phys.Rev. D 75, 064008 (2007).

[38] R.G. Cai and Y.S. Myung, Phys. Rev. D 67, 124021 (2003). 
[39] B. Wang, E. Abdalla and R.K. Su, Phys. Lett. B 503, 394 (2001).

[40] B. Wang, E. Abdalla and R.K. Su, Mod. Phys. Lett. A 17, 23 (2002).

[41] E. Verlinde, JHEP. 1104, 029 (2011).

[42] J. Zhou, B. Wang, Y. Gong, E. Abdalla, Phys. Lett. B 652, 86 (2007).

[43] A. Sheykhi, B. Wang, Phys. Lett. B 678, 434 (2009).

[44] A. Sheykhi, B. Wang, Mod. Phys. Lett. A 25, 1199 (2010).

[45] S. Das, P. Majumdar and R. K. Bhaduri, Class. Quantum Grav. 19, 2355 (2002).

[46] B. Saha, Astrophys. Space Sci. 302, 83 (2006).

[47] A. Pradhan, S.K. Singh, Int. J. Mod. Phys. D 13, 503 (2004).

[48] B. Saha, Int. J. Theor. Phys. 45, 983 (2006).

[49] P. Aluri et al., J. Cosmol. Astropart. Phys. 12, 3 (2013).

[50] V. Fayaz, H. Hossienkhani, A. Pasqua, Z. Zarei, and M. Ganji, Can. J. Phys. 94, 1 (2016).

[51] H. Hossienkhani, A. Najafi and N. Azimi, Astrophys. Space Sci. 353, 311 (2014).

[52] H. Hossienkhani and A. Pasqua, Astrophys. Space. Sci. 349, 39 (2014); Kh. Saaidi and H. Hossienkhani, Astrophys. Space. Sci. 333, 305 (2011); V. Fayaz, H. Hossienkhani, Astrophys. Space. Sci. 343, 291 (2013); V. Fayaz, M.R. Setare and H. Hossienkhani, Can. J. Phys. 91, 153 (2013).

[53] H.A. Borges, S. Carneiro, Gen. Rel. Grav. 37, 1385 (2005).

[54] L. Amendola, Phys. Rev. D 62, 043511 (2000); L. Amendola and C. Quercellini, Phys. Rev. D 68, 023514 (2003); L. Amendola, S. Tsujikawa and M. Sami, Phys. Lett. B 632, 155 (2006).

[55] D. Pavon, W. Zimdahl, Phys. Lett. B 628, 206 (2005).

[56] C.G. Boehmer, G. Caldera-Cabral, R. Lazkoz, R. Maartens, Phys. Rev. D 78, 023505 (2008).

[57] G. Boehmer, F. Atrio-Barandela and D. Pavon, Phys. Rev. D 74, 043521 (2006).

[58] O. Bertolami, F. Gil Pedro and M. Le Delliou, Phys. Lett. B 654, 165 (2007).

[59] R.G. Cai and S.P. Kim, JHEP 0502, 050 (2005).

[60] J. Zhang, Phys. Lett. B 668, 353 (2008). 